Hongyan Li, Limeng Song, Cong Sun, Ruyi Li, Yongqiang Fu, Hongjie Zhang, Aiwu Yang and Hongli Liu*

\title{
Thermal insulation of silica aerogel/ PMMA composites with amino-capped polydivinylsiloxane phase interfaces
}

https://doi.org/10.1515/secm-2017-0248

Received July 24, 2017; accepted October 18, 2017; previously published online February 28, 2018

\begin{abstract}
Contradictions of strength and thermal insulation of silica aerogel (SA) are of wide concern. In this research, the mesoporous structure of SA was grafted with the polymers to increase the strength of SA and decrease the thermal conductivity of composites. The composites were characterized and analyzed using field emission scanning electron microscopy (SEM), Fourier transform infrared (FTIR) spectroscopy, the thermal gravimetric analyzer (TGA), multi-assay gel permeation chromatography (GPC), a microcomputer control electronic universal testing machine and so on. The results indicated that amino-capped polydivinylsiloxane (AP) was successfully grafted on the SA surface and polymerized with methyl methacrylate (MMA) to form an AP high thermal insulation phase interface between the SA phase and poly (methyl methacrylate) (PMMA) phase. The SA-AP/PMMA composite with a covalent bonding high thermal insulation phase interface had better mechanical properties and compressive strength than those of SA/ PMMA. The obtained composite was an excellent thermal insulation material with lower thermal conductivity $[0.045 \mathrm{~W} /(\mathrm{m} \cdot \mathrm{K})]$.
\end{abstract}

\footnotetext{
*Corresponding author: Hongli Liu, School of Materials Science and Engineering, Tianjin Chengjian University, Tianjin 300384, P.R. China, e-mail: Ihlbh@163.com; and Tianjin Key Laboratory of Soft Soil Characteristics and Engineering Environment, Tianjin 300384, P.R. China

Hongyan Li: School of Materials Science and Engineering, Tianjin Chengjian University, Tianjin 300384, P.R. China; and Tianjin Key Laboratory of Soft Soil Characteristics and Engineering Environment, Tianjin 300384, P.R. China

Limeng Song, Cong Sun, Ruyi Li, Yongqiang Fu and Hongjie Zhang: School of Materials Science and Engineering, Tianjin Chengjian University, Tianjin 300384, P.R. China

Aiwu Yang: School of Civil Engineering, Tianjin Chengjian University, Tianjin 300384, P.R. China; and Tianjin Key Laboratory of Soft Soil Characteristics and Engineering Environment, Tianjin 300384, P.R. China
}

Keywords: compressive strength; high thermal insulation phase interface; silica aerogel; thermal conductivity.

\section{Introduction}

Aerogels were discovered in the 1930s by Kistler. Silica aerogel (SA) was prepared firstly with the methodology of substituting the liquid phase of the gel via gas with only a minimal shrinkage of the gel [1]. SAs are a low-density, translucent and thermal insulating material consisting of nanoparticle building blocks, networked together to form an open, highly porous structure [2]. They have large specific surface area $\left(500-1500 \mathrm{~m}^{2} / \mathrm{g}\right)$, high porosity (80-99\%), and low bulk density $\left(0.03-0.35 \mathrm{~g} / \mathrm{cm}^{3}\right)$ [3-5]. SAs belong to the largest class of highly porous inorganic aerogels [6-8]. According to the International Union of Pure and Applied Chemistry (IUPAC) recommendation [9, 10], porous materials can be categorized into three groups on account of their pore diameter: microporous (pore diameter $<2 \mathrm{~nm}$ ), mesoporous (pore diameter ranges from $2 \mathrm{~nm}$ to $50 \mathrm{~nm}$ ), and macroporous (pore diameter more than $50 \mathrm{~nm}$ ) [11, 12]. Moreover, nanoporous implies pore diameter $<100 \mathrm{~nm}[13,14]$. Pure SAs are brittle and feature poor mechanical properties [15].

Thus different types of organics are usually added in the SAs as reinforcing phase [16-18] to enhance their mechanical properties, such as poly(methyl methacrylate) (PMMA) [19, 20], PVC [21, 22] and KH-570 [23]. A review of literature indicates that some studies have been done on the synthesis and mechanical reinforcing strategies of SA/polymer nanocomposites [24]. Boday et al. [25] reported that the optimal mechanical reinforcement of aerogels could be achieved by grafting higher molecular weight organic macromolecules onto $\mathrm{SiO}_{2}$ surfaces. Density of the material was not significantly increasing. The well-defined polystyrene/SA nanocomposites were synthesized through reversible additionfragmentation chain transfer polymerization by Sobani et al. [26]. In their report, the polystyrene was attached on SA surface by using double bonds containing modifier 
(surface modification) [27]. Although it has been proved that compounding of silica backbone with chemically bonded polymer is an effective way to increase mechanical strength, whether the thermal conductivity of aerogels would increase is still unknown. Research by Losego et al. [28], Losego and Cahill [29] and Nitzan [30] shows that the strength of covalent chemical bonds directly controls phonon heat transport across an interface [31-33]. The interfacial conductivity increases about $80 \%$ from Van der Waals to covalent bonding.

In this research, the $\mathrm{SA} /$ polymer composites were prepared via the sol-gel $[34,35]$ method through ambient pressure drying $[36,37]$ technology. Amino-capped polydivinylsiloxane (AP) as a high thermal insulation phase interface [38-44] was constructed between the silica phase and PMMA phase, and the heat flux could be held in the silica phase. This was to achieve as great a decrease in overall thermal conductivity of the material as possible. The microstructure, mechanical properties, thermal conductivity, and thermal stability of the composites were analyzed. In addition, the high thermal insulation phase interfaces of SA-AP/PMMA were studied. The construction of phase interfaces and the mechanisms of phase interfaces on the reinforcement and thermal conduction of the composites were investigated.

\section{Materials and methods}

\subsection{Materials}

AP (analytical grade) was purchased from Dow Corning Corporation (Midland, MI, USA). Benzoyl peroxide (analytical grade) was obtained from Chengdu Kelong Chemical Reagent Factory (Chengdu, China). Other reagents were all of analytical grade.

\subsection{Preparation of materials}

The precursor solution was obtained by mixing tetraethyl orthosilicate, ethanol and deionized water with a molar ratio of 1:8:3.75, respectively. Hydrochloric acid was added into the precursor solution, $\mathrm{pH}$ value was adjusted to 3-4, and the mixture was stirred for $60 \mathrm{~min}$ to make it evenly mixed. The mixture solution was hydrolyzed in constant temperature for $16 \mathrm{~h}$, then $0.5 \mathrm{~mol} / \mathrm{l}$ ammonia solution was added to adjust the $\mathrm{pH}$ value to $7-8$. The mixture was stirred until a gel formed, ethanol solution was added and aged for $12 \mathrm{~h}$. Then wet gels of $\mathrm{SiO}_{2}$ were obtained through solvent replacement in 2 days (ethanol one time and n-hexane twice), and SA could be obtained through ambient pressure drying. The wet gels of $\mathrm{SiO}_{2}$ were modified by $\mathrm{AP}$, and the composite (SA-AP) was washed in n-hexane in order to remove the residual AP liquid. The SA-AP was prepared after drying at $50^{\circ} \mathrm{C}(4 \mathrm{~h}), 80^{\circ} \mathrm{C}(3 \mathrm{~h})$ and $120^{\circ} \mathrm{C}$ $(2 \mathrm{~h})$, respectively. Five parts of $0.06 \mathrm{~g}$ benzoyl peroxide, $15 \mathrm{ml}$ MMA were accurately weighed and 3\%, 4\%, 5\%, 6\% and $7 \%$ of SA-AP were added into the system, respectively. The system was stirred, the temperature was heated up to $85^{\circ} \mathrm{C}$, kept for $45 \mathrm{~min}$ until the prepolymer became sticky, and controlled quickly to a temperature at about $40^{\circ} \mathrm{C}$ with cooling water. The SA-AP/PMMA composite aerogels materials were obtained by allowing the prepolymer to stand until all the bubbles were lifted. The aerogels were heated at $50^{\circ} \mathrm{C}$ for $2 \mathrm{~h}$ and then at $70^{\circ} \mathrm{C}$ for $2 \mathrm{~h}$.

\subsection{Characterization}

The pore size distribution and morphology of SAs were observed by using field emission scanning electron microscopy (SEM) (NanoSEM430, FEI Company, USA). Determination of the materials of the groups was via Fourier transform infrared (FTIR) spectroscopy (Thermo Nicolet 380, Thermoelectri Corporation, USA). The composition of the materials was qualitatively analyzed by using a synchronous thermal analyzer (DTU-2B, TG, Beijing Boyuan Precision Technology Development Corporation). The molecular weight and molecular weight distribution of polymers were tested by using a multiassay gel chromatograph (TDA305, GPC, Malvern, UK). The compressive strength of the samples was tested using a microcomputer control electronic universal testing machine (CMT6104, Shenzhen Xinsansi Material Testing Corporation), with the implementation of ISO 604-2002 as standard. Diameter (d) of the samples was measured with a Vernier caliper, and the average of three measurements was taken (accurate to $0.02 \mathrm{~mm}$ ). The thermal conductivity of solid materials was measured by the transient hot wire method (TC-3000E, Xian Xiaxi Electronic Technology Corporation), and the average of three measurements was obtained at $33.05^{\circ} \mathrm{C}$, with acquisition time of $5 \mathrm{~s}$, and test voltage $1.50 \mathrm{~V}$.

\section{Results and discussion}

Figure 1 shows the photographs of the SA and composite aerogels. Usually, the $\mathrm{SiO}_{2}$ aerogel monolith is not easy 


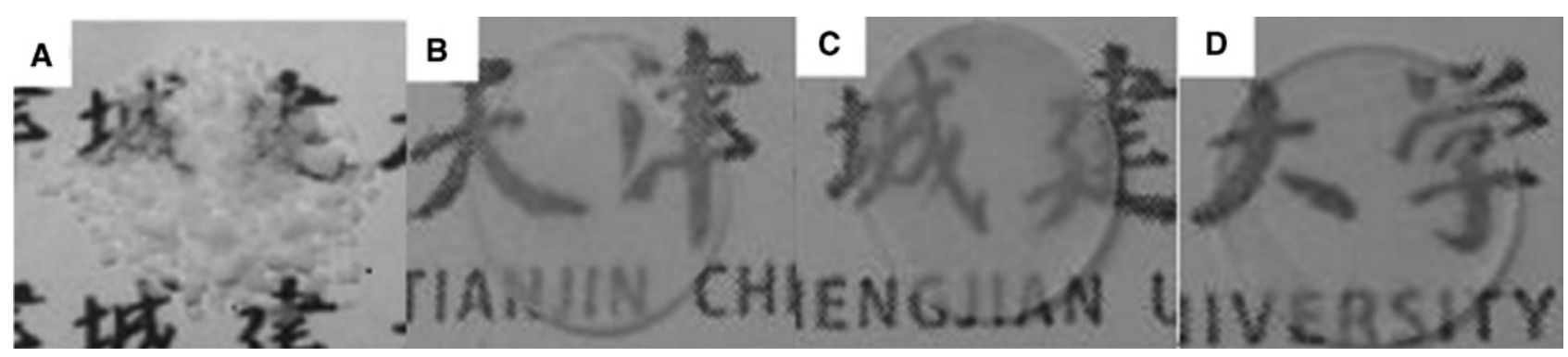

Figure 1: Photographs of (A) silica aerogel (SA), (B) SA-poly(methyl methacrylate) (PMMA), (C) SA-KH570/PMMA, (D) SA-amino-capped polydivinylsiloxane (AP)/PMMA.

to prepare through ambient pressure drying, because the bonding force of $\mathrm{SiO}_{2}$ nanoparticles is fragile which decreases the strength of the $\mathrm{SiO}_{2}$ wet gel skeleton. Thus, the polymers are introduced to improve the strength of SA. As expected, the strength of SA/PMMA, SA-KH570/PMMA and SA-AP/PMMA composite aerogels are all improved by adding polymers. Differences between the three composite aerogels are not observed besides transparency.

Evaluation of size distribution and surface morphology of the modified SA three-dimensional network structure was performed by SEM. Figure 2 exhibits SEM images of SA, SA/PMMA, SA-KH570/PMMA and SA-AP/ PMMA. According to the images, $\mathrm{SiO}_{2}$ nanoparticles are spherical shape. Also, as expected, pure SA (Figure 2A) is spongy. It was formed from silica nanoparticles with diameter around $20 \mathrm{~nm}$. Compact aggregated spherical nanoparticles with similar size of $10-20 \mathrm{~nm}$ are observed (Figure 2B). This is because there is no phase interface between the SA phase and PMMA phase. Therefore, PMMA could not be coated on the surface of SA effectively.
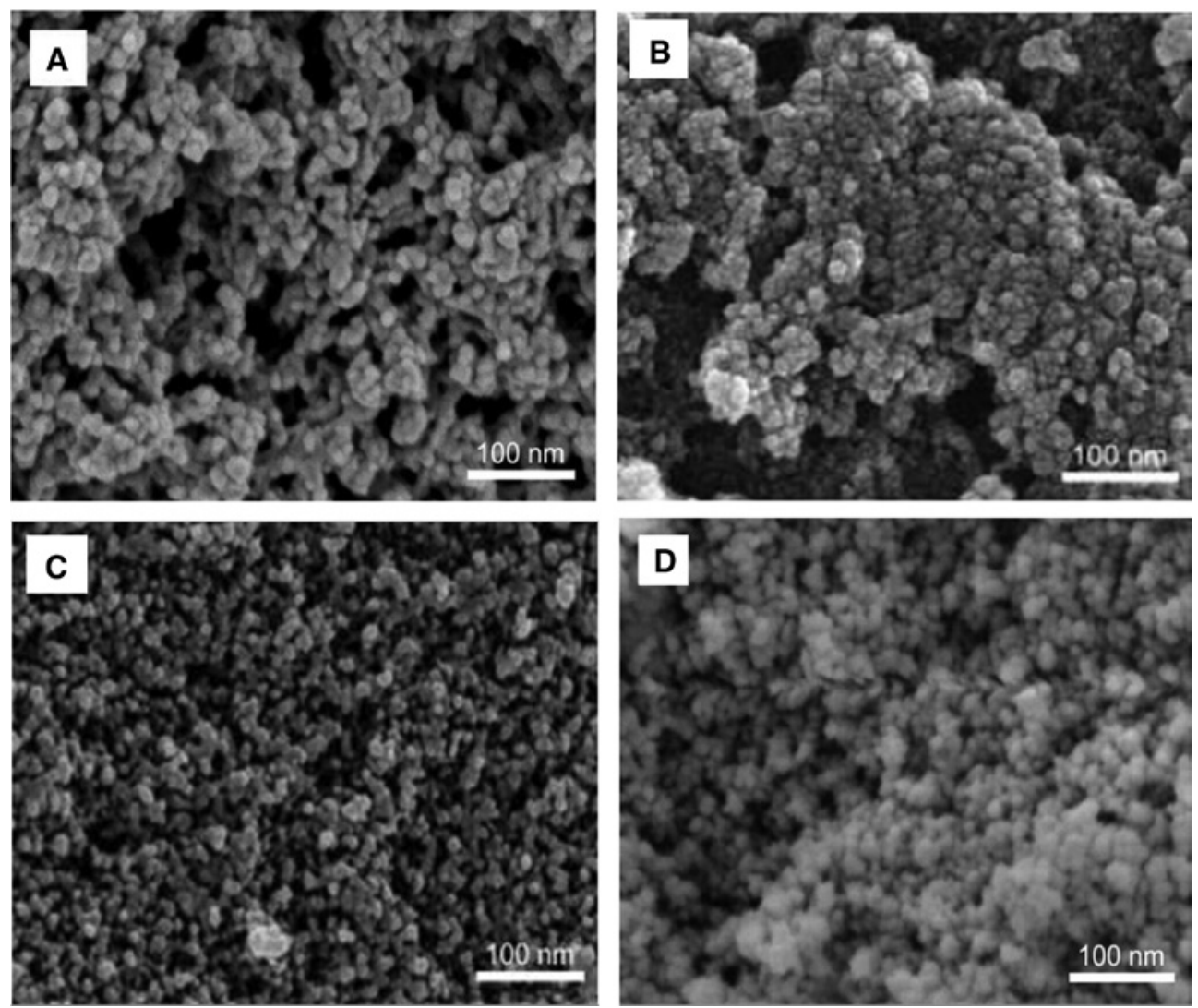

Figure 2: Scanning electron microscopy (SEM) images of (A) silica aerogel (SA), (B) SA/poly(methyl methacrylate) (PMMA), (C) SA-KH570/ PMMA and (D) SA-amino-capped polydivinylsiloxane (AP)/PMMA. 
Figure 2C exhibits a honeycomb porous structure with fine particulate morphology; the structure is quite uniform, and contains $10-15 \mathrm{~nm}$ silica clusters. Figure 2D depicts SEM images of SA-AP/PMMA. The images confirm a spongy structure of the composite.

Nitrogen adsorption/desorption isotherms of the SA and composites are represented in Figure 3. Tests concerning pore size and surface area were conducted and the results are shown in Table 1. All the curves corresponding to the samples belong to IV isotherm with a type H2 hysteresis loop, according to the IUPAC classification $[9,10]$, which is characteristic of a mesoporous structure with cylindrical pores. The $\mathrm{N}_{2}$ desorption cycle of the isotherm showed a hysteresis loop which can be attributed to the capillary condensation occurring in the mesoporous materials. The specific surface areas of the SA, SA/PMMA, SA-KH570/PMMA and SA-AP/PMMA were calculated as $529.98 \mathrm{~m}^{2} / \mathrm{g}, 184.87 \mathrm{~m}^{2} / \mathrm{g}, 501.05 \mathrm{~m}^{2} / \mathrm{g}$ and $639.04 \mathrm{~m}^{2} / \mathrm{g}$, respectively. Average pore diameters were estimated at around $13.07 \mathrm{~nm}, 23.77 \mathrm{~nm}, 14.18 \mathrm{~nm}$ and $11.42 \mathrm{~nm}$, respectively. According to the data in Table 1, it is evident that the specific surface areas increase with decreasing pore size, which is due to the structural adjustment. It is noted

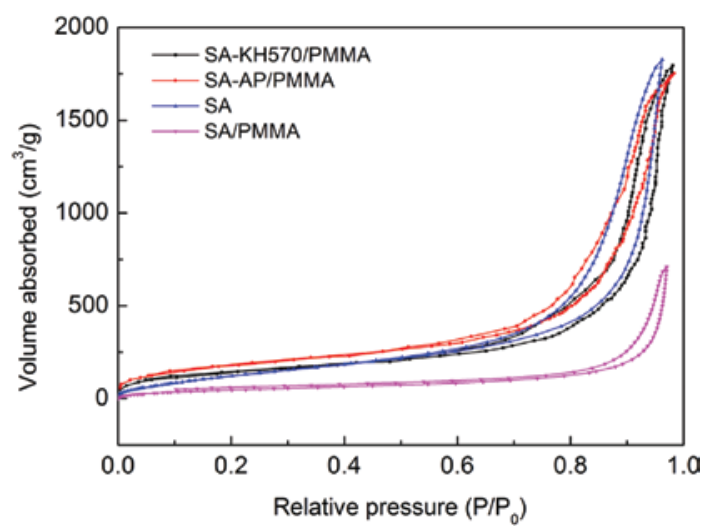

Figure 3: $N_{2}$ adsorption/desorption isotherms of silica aerogel (SA) and composites.

Table 1: Specific surface area and average pore diameter data of silica aerogel (SA) and composites.

\begin{tabular}{lrr}
\hline Sample & $\begin{array}{r}\text { Specific surface } \\
\text { area }\left(\mathbf{m}^{2} / \mathbf{g}\right)\end{array}$ & $\begin{array}{r}\text { Average pore } \\
\text { diameter }(\mathbf{n m})\end{array}$ \\
\hline SA & 529.98 & 13.07 \\
SA/PMMA & 184.87 & 23.77 \\
SA-KH570/PMMA & 501.05 & 14.18 \\
SA-AP/PMMA & 639.04 & 11.42 \\
\hline
\end{tabular}

AP, Amino-capped polydivinylsiloxane; PMMA, poly(methyl methacrylate); SA, silica aerogel. here that SA-AP/PMMA has a larger BET specific surface area $\left(639.04 \mathrm{~m}^{2} / \mathrm{g}\right)$ than that of the other three samples. Moreover, it can be seen that the pore structure of SA-AP/ PMMA compared with the SA and SA-KH570/PMMA has no obvious changes through the SEM images and nitrogen adsorption/desorption isotherm. However, the specific surface areas and pore structure of SA/PMMA are obviously different from those of the other three samples, which may be due to the lower porosity. These results are consistent with the results of SEM analysis.

The FTIR spectra of SA, PMMA, SA/PMMA, SA/AP and SA-AP/PMMA are illustrated in Figure 4A-E. As shown in Figure 4A, the absorption peak appearing at $1082 \mathrm{~cm}^{-1}$ is relative to the stretching vibration of Si-O. The peak at about $3450 \mathrm{~cm}^{-1}$ is associated with the $-\mathrm{OH}$ group on the surface of the SA sample. The broad peak in the range of around $1648 \mathrm{~cm}^{-1}$ is due to the absorbed water molecules. The spectrum of PMMA (Figure 4B) absorption peak appears at $1740 \mathrm{~cm}^{-1}$ because of the stretching vibration of ester groups $(\mathrm{C}=0)$. The peaks at $2954 \mathrm{~cm}^{-1}$ and $2997 \mathrm{~cm}^{-1}$ are relative to stretching vibrations of SP3 and SP2 hybrid hydrogen bonds of C-H groups, respectively. The intense peak at $1100 \mathrm{~cm}^{-1}$ is the characteristic peak for C-O-C. Figure 3C shows intense absorption bands at about $1730 \mathrm{~cm}^{-1}$ because of the stretching vibration of $\mathrm{C}=0$ groups. The peak at $1148 \mathrm{~cm}^{-1}$ is relative to the stretching vibration of $\mathrm{C}-\mathrm{O}-\mathrm{C}$ groups. Absorption peaks appearing at $754 \mathrm{~cm}^{-1}$ and $844 \mathrm{~cm}^{-1}$ are associated with bending vibration of Si-C. It is obvious that in the spectrum of the SA-AP (Figure 4D), the absorption peak showing at $2964 \mathrm{~cm}^{-1}$ is related to stretched vibration of $\mathrm{C}-\mathrm{H}$ with the unsaturated olefins' carbon atoms. The peak at around $2361 \mathrm{~cm}^{-1}$ is ascribed to existing tertiary amine salt $\left(\mathrm{NH}^{+}\right)$. The absorption peak appearing at $1263 \mathrm{~cm}^{-1}$ is associated with

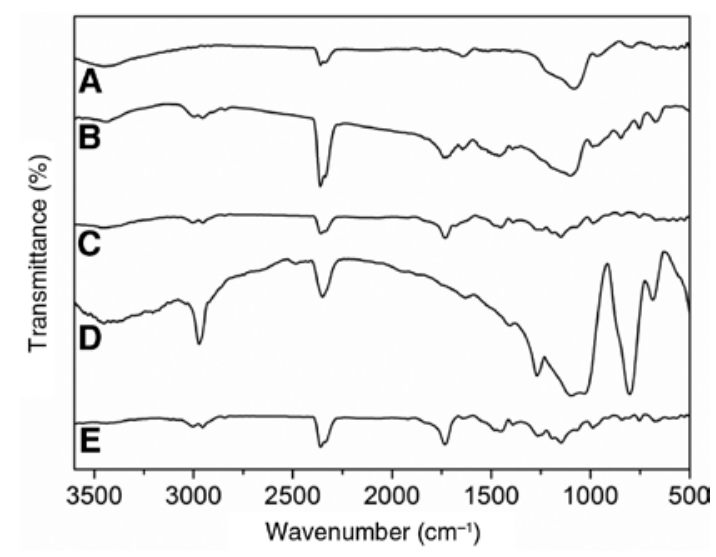

Figure 4: Fourier transform infrared (FTIR) spectra of (A) silica aerogel (SA), (B) poly(methyl methacrylate) (PMMA), (C) SA/PMMA, (D) SA/amino-capped polydivinylsiloxane (AP) and (E) SA-AP/PMMA. 
bending vibration of $\mathrm{C}-\mathrm{O}$. A sharp absorption peak appears at $1086 \mathrm{~cm}^{-1}$ due to the stretching vibration of Si-O-Si and the peak at $804 \mathrm{~cm}^{-1}$ is ascribed to the $\mathrm{Si}-\mathrm{C}$ groups. As shown in Figure 4E, the absorption peak showing at $2950 \mathrm{~cm}^{-1}$ is associated with stretching vibration of C-H with the unsaturated olefins' carbon atoms. The peak at around $2360 \mathrm{~cm}^{-1}$ is attributed to existing tertiary amine salt $\left(\mathrm{NH}^{+}\right)$. An absorption peak appears at about $1740 \mathrm{~cm}^{-1}$ because of the stretching vibration of $\mathrm{C}=0$ groups. The peaks at about $1268 \mathrm{~cm}^{-1}$ and $1145 \mathrm{~cm}^{-1}$ are relative to the stretching vibration of $\mathrm{C}-\mathrm{O}-\mathrm{C}$ groups and this showed that exit ester groups. The above results demonstrated that the high thermal insulation phase interfaces between SA and PMMA were obtained by the covalent chemical bonds.

Figure 5 shows TGA curves of SA, PMMA, SA/AP, SA/ PMMA, SA-KH570/PMMA and SA-AP/PMMA samples at a heating rate of $20^{\circ} \mathrm{C} / \mathrm{min}$. The SA was heated to $600^{\circ} \mathrm{C}$ under the air atmosphere from room temperature. The approximate straight line (Figure 5) showed that no distinct change is observed in weight. The weight of the SA/ AP sample decreased $<5 \%$ when the temperature continuously increased to $375^{\circ} \mathrm{C}$ in the air atmosphere. The weight loss can be attributed to the evaporation of water molecules, volatilization of residual organic monomers and degradation of low molecular weight oligomers which were absorbed by $\mathrm{OH}$ groups on the surface of the SA nanoparticles. The weight decreased $30 \%$ when the temperature increased from $375^{\circ} \mathrm{C}$ to $494^{\circ} \mathrm{C}$ because of AP decomposed and organic groups being removed. The weight decreased $17.5 \%$ when the temperature increased from $494^{\circ} \mathrm{C}$ to $540^{\circ} \mathrm{C}$. The weight loss can demonstrate that the majority of the AP continuously decomposed and organic groups were continually removed. There was almost no change at all in the weight of samples when the temperature increased more than $540^{\circ} \mathrm{C}$ due to the AP

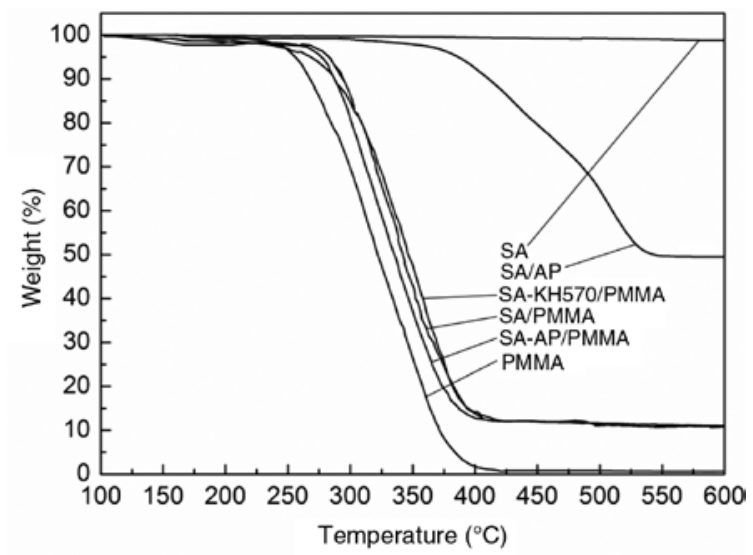

Figure 5: TGA curves of polymer and composites.
Table 2: TGA data of poly(methyl methacrylate) (PMMA) and composites.

\begin{tabular}{lcc}
\hline Sample & $\mathrm{T}_{0}\left({ }^{\circ} \mathrm{C}\right)$ & $\mathrm{T}_{50}\left({ }^{\circ} \mathrm{C}\right)$ \\
\hline PMMA & 251.2 & 321.5 \\
SA/AP & 370.1 & 473.4 \\
SA/PMMA & 270.6 & 341.5 \\
SA-KH570/PMMA & 286.4 & 346.8 \\
SA-AP/PMMA & 267.3 & 333.7 \\
\hline
\end{tabular}

AP, Amino-capped polydivinylsiloxane; PMMA, poly(methyl methacrylate); SA, silica aerogel.

having already wholly decomposed. The results indicated that AP was successfully grafted onto the surface of SA.

According to the TGA results, the onset temperature $\left(\mathrm{T}_{0}\right)$ and temperature at a weight loss of $50 \%\left(\mathrm{~T}_{50}\right)$ of the samples are summarized in Table $2 . \mathrm{T}_{0}$ and $\mathrm{T}_{50}$ of samples are typical parameters for reflecting the thermal stability of them. The $\mathrm{T}_{50}$ values of PMMA, SA/AP, SA/PMMA, SA-KH570/PMMA and SA-AP/PMMA correspond to $321.5^{\circ} \mathrm{C}$, $473.4^{\circ} \mathrm{C}, 341.5^{\circ} \mathrm{C}, 346.9^{\circ} \mathrm{C}$ and $333.7^{\circ} \mathrm{C}$, respectively. The $\mathrm{T}_{50}$ values indicated that thermal stabilities of the composite samples are higher than that of PMMA. The reason could be incorporation into the SA phase, which consists of $\mathrm{SiO}_{2}$ particles. In addition, slight increases in degradation temperatures were obtained because of this inorganic 3D skeleton having a high thermal stability. Thus, thermal stabilities of the samples were improved by adding the $\mathrm{SA}$ component. It can be observed that the $\mathrm{T}_{50}$ value of SA-KH570/PMMA is higher than that of SA/PMMA, which could mean a mild improvement in thermal stability. As we know, thermal stability of a material is related to its thermal conductivity to some extent [2]. The SA-KH570/ PMMA composite has a covalent bond linked interface between SA and polymer phases. This leads to a more efficient path for thermal conductivity. Therefore, thermal conductivity of the SA-KH570/PMMA composite was improved. It was easy to get a balance in the heat flux in materials in a high temperature environment. Local high temperature zones in this composite could be avoided, and the thermal stability would increase. The $T_{50}$ values of the SA-AP/PMMA sample are the lowest among all the composites. This could be attributed to high thermal insulation phase interfaces between the SA and PMMA.

Figure 6 shows the graph of molecular weight of PMMA, AP/PMMA and SA-AP/PMMA*. AP/PMMA represents that the copolymer of MMA and AP was synthesized. SA-AP/PMMA ${ }^{\star}$ denotes that the copolymer of MMA and AP was grafted on the surface of SA. As shown in Figure 5, the number average molecular weights of the PMMA, $\mathrm{AP} / \mathrm{PMMA}$ and SA-AP/PMMA ${ }^{*}$ are $27.9 \times 10^{4}, 27.7 \times 10^{4}$ and 


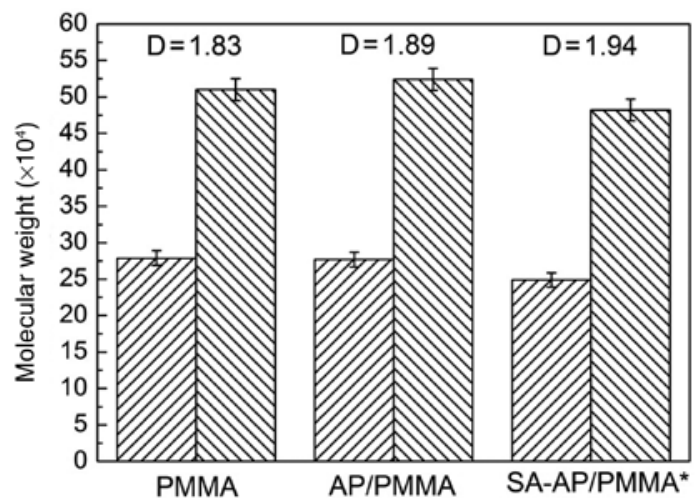

Figure 6: Molecular weight of polymer and composites.

$24.9 \times 10^{4}$, respectively. The number average molecular weights of the PMMA and PMMA/AP are larger than that of SA-AP/PMMA*. This shows that MMA radical polymerization was inhibited by adding SA and the number average molecular weight decreased. The weight average molecular weights of PMMA, AP/PMMA and SA-AP/PMMA* are $51 \times 10^{4}, 52.4 \times 10^{4}$ and $48.2 \times 10^{4}$, respectively. The molecular weight of polymer is polydisperse, and the dispersibility is often expressed by $\mathrm{D}$. The larger the $\mathrm{D}$ was, the wider the molecular weight distribution. Figure 6 shows that the dispersion coefficients of the PMMA, AP/PMMA and SA-AP/PMMA* are 1.83, 1.89 and 1.94, respectively. A number of different molecular chain lengths of the homologue of polymer were synthesized via introducing SA. Thus, the dispersion of SA-AP/PMMA* is larger than that of the other samples.

Figures 7 and 8 exhibit the compressive strength of SA, PMMA, SA/PMMA and SA-AP/PMMA. As shown

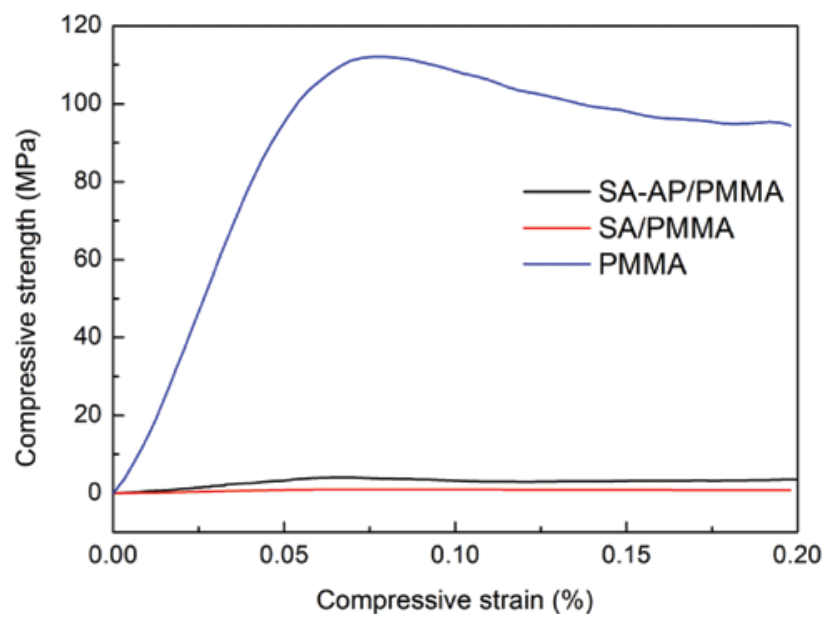

Figure 7: Compressive strength of poly(methyl methacrylate) (PMMA), silica aerogel (SA)/PMMA and SA-amino-capped polydivinylsiloxane (AP)/PMMA.

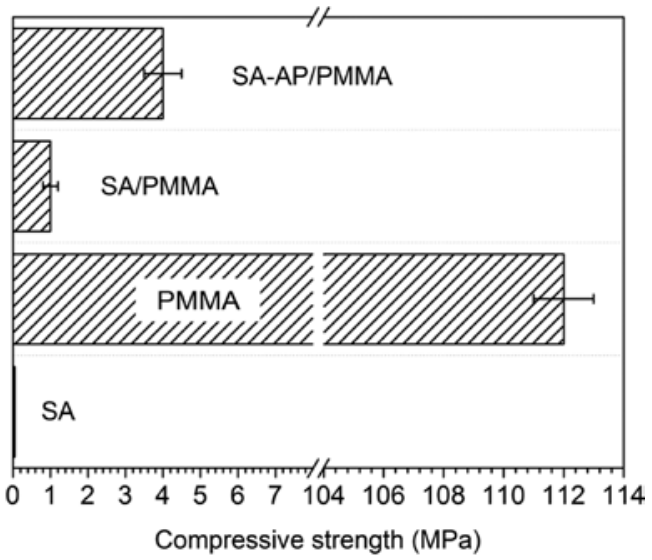

Figure 8: Mechanical properties of silica aerogel (SA), poly(methyl methacrylate) (PMMA), and composites.

in Figure 7, the compressive strength of SA is too weak to detect. The pure PMMA sample had a compressive strength of around $112 \mathrm{MPa}$. The compressive strength of the composites increased with the increase in PMMA and AP content, and the largest compressive strength of SA/ PMMA was around $1 \mathrm{MPa}$ with $5 \%$ SA component. It is obvious that the SA-AP/PMMA sample with 5\% SA content had the largest compressive strength of around $4 \mathrm{MPa}$. This could be related to the AP grafted on the surface of SA generated flexible phase interfaces with PMMA, which is an efficient load transfer media. The PMMA and SA formed a monolithic through the flexible phase interfaces. The properties of compatible and interface adhesion were improved. The compressive strength of composite materials was increased due to cracks propagating slowly and stress dispersing easily. The materials compressive strength was improved through the surface of SA loaded polymers with uniform distribution.

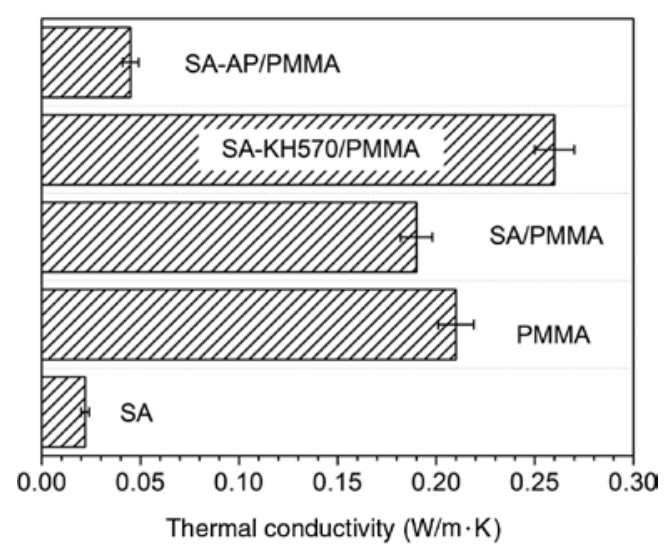

Figure 9: Thermal conductivity of silica aerogel (SA), poly(methyl methacrylate) (PMMA), and composites. 
Figure 9 shows thermal conductivity of SA, PMMA, SA/PMMA, SA-KH570/PMMA and SA-AP/PMMA. As shown in Figure 9, the SA sample had a thermal conductivity of about $0.022 \mathrm{~W} /(\mathrm{m} \cdot \mathrm{K})$, which is a super thermal insulation material. The thermal conductivity of PMMA was about $0.21 \mathrm{~W} /(\mathrm{m} \cdot \mathrm{K})$, and the thermal conductivity of SA/PMMA was around $0.19 \mathrm{~W} /(\mathrm{m} \cdot \mathrm{K})$, which is smaller than that of pure PMMA. This should be ascribed to the incorporation of SA with a higher thermal insulation. The thermal conductivity of SA-KH570/PMMA with covalent bonding phase interfaces was much larger than that of the other samples, and was about $0.26 \mathrm{~W} /(\mathrm{m} \cdot \mathrm{K})$. The thermal conductivity of SA-AP/PMMA with a low value is only $0.045 \mathrm{~W} /(\mathrm{m} \cdot \mathrm{K})$. This would be relative to the incorporation of AP monomer to form flexible phase interfaces with a higher thermal insulation.

\section{Conclusion}

Composite aerogels with polymers as reinforcement were successfully prepared through the sol-gel method combined with ambient pressure drying. Aerogels were characterized by SEM and BET surface area analyzer. The results indicated that SA-AP/PMMA has a porous and spongy structure and spherical shape with a diameter of around $20 \mathrm{~nm}$. The SA-AP/PMMA specific surface area was as large as $639.04 \mathrm{~m}^{2} / \mathrm{g}$, which was even larger than that of the SA, SA/PMMA and SA-KH570/PMMA. The average pore diameter of SA-AP/PMMA (11.42 nm) was lower than that of SA $(13.07 \mathrm{~nm})$, SA/PMMA $(23.77 \mathrm{~nm})$ and SA-KH570/PMMA (14.18 nm) samples. FTIR spectroscopy results showed that AP was successfully grafted to the SA surface and polymerized with MMA to form a high thermal insulation phase interface. The SA-AP/ PMMA composite with covalent bonding phase interface had good mechanical properties. The compressive strength of SA-AP/PMMA (4 MPa) was larger than that of SA/PMMA (1 MPa). The thermal conductivity of the SA-AP/PMMA was $0.045 \mathrm{~W} /(\mathrm{m} \cdot \mathrm{K})$, which was obviously lower than the thermal conductivity of the PMMA $[0.21 \mathrm{~W} /(\mathrm{m} \cdot \mathrm{K})], \mathrm{SA} / \mathrm{PMMA}[0.19 \mathrm{~W} /(\mathrm{m} \cdot \mathrm{K})]$ and SA-KH570/PMMA $[0.26 \mathrm{~W} /(\mathrm{m} \cdot \mathrm{K})]$. Based on these results, it can be confirmed that a high thermal insulation phase interface between SA and PMMA was formed and it was strong enough for supporting the SA skeleton. The thermal insulation of the SA-AP/PMMA composite was also found to be more excellent than SA/PMMA and SA-KH570/PMMA, which could be related to the flexible phase interfaces with high thermal insulation.
Acknowledgments: This work was financially supported by the National Natural Science Foundation of China (Funder Id: 10.13039/501100001809, Grant 51503141 and 51472175)

\section{References}

[1] Kistler SS. Nature 1931, 127, 741-741.

[2] Li HY, Song LM, Fu YQ, Wei YF, Li RY, Liu HL. Compos. Sci. Technol. 2017, 138, 169-178.

[3] Mulik S, Sotiriou-Leventis C, Churu G, Lu H, Leventis N. Chem. Mater. 2008, 20, 5035-5046.

[4] Rao AV, Pajonk GM. J. Non-Cryst. Solids 2001, 285, 202-209.

[5] Dai S, Ju YH, Gao HJ, Lin JS, Pennycook SJ, Barnes CE. Chem. Commun. 2000, 3, 243-244.

[6] Jiang YG, Feng JZ, Feng J. J. Sol-Gel Sci. Technol. 2017, 83, 64-71.

[7] Liu H, Xia XL, Ai Q, Xie XQ, Sun C. Exp. Therm. Fluid Sci. 2017, 84, 67-77.

[8] Wu XD, Shao GF, Liu SJ, Shen XD, Cui S, Chen XB. Powder Technol. 2017, 312, 1-10.

[9] Mirshafiei-Langari SA, Haddadi-Asl V, Roghani-Mamaqani H, Sobani M, Khezri K. J. Polym. Res. 2013, 20, 163-174.

[10] Xi J, Qiu X, Zhu W, Tang X. Microporous Mesoporous Mater. 2006, 88, 1-7.

[11] Tao Y, Endo M, Kaneko K. Recent Pat. Chem. Eng. 2008, 1, 192-200.

[12] Cabanas A, Enciso E, Carbajo MC, Torralvo MJ, Pando C, Renuncio JAR. Chem. Mater. 2005, 17, 6137-6145.

[13] Logar NZ, Kau V. Acta Chim. Slov. 2006, 53, 117-135.

[14] Deng Z, Wei J, Xue X, Wang J, Chen L. J. Porous Mater. 2001, 8 , 37-42.

[15] Moner-Girona MM, Martinez E, Roig A, Esteve J, Molins E. J. Non-Cryst. Solids 2001, 285, 244-250.

[16] Gangopadhyay R, De A. Chem. Mater. 2000, 12, 608-622.

[17] Burkett SL, Ko N, Stern ND, Caissie JA, Sengupta D. Chem. Mater. 2006, 18, 5137-5143.

[18] Zou H, Wu S, Shen J. Chem. Rev. 2008, 108, 3893-3957.

[19] Guo JB, Zhang DH, Shao HJ, Zhang KZ, Wu B. Sci. Eng. Compos. Mater. 2015, 22, 17-23.

[20] Yang BH, Li M, Wu Y, Huang JJ, Zhang H, Deng CH. Sci. Eng. Compos. Mater. 2013, 20, 147-153.

[21] Jabbari M, Akesson D, Skrifvars M, Taherzadeh MJ. J. Reinf. Plast. Comp. 2015, 34, 1581-1592.

[22] Mujal-Rosas R, Marin-Genesca M, Ballart-Prunell J. Sci. Eng. Compos. Mater. 2015, 22, 231-243.

[23] Yasir Ali A, Jasim A, Abdulsalam AH, Harsha J, Antony J. Int. J. Biol. Macromol. 2017, 101, 1041-1050.

[24] Maleki H, Duraes L, Portugal A. J. Non-Cryst. Solids 2014, 385, 55-74.

[25] Boday DJ, Keng PY, Muriithi B, Pyun J, Loy DA. J. Mater. Chem. 2010, 20, 6863-6865.

[26] Sobani M, Haddadi-AsI V, Mirshafiei-Langari S, Salami-Kalajahi M, Roghani-Mamaqani H, Khezri K. Des. Monomers Polym. 2014, 17, 245-254.

[27] Sobani M, Haddadi-AsI V, Salami-Kalajahi M, RoghaniMamaqani H. J. Sol-Gel Sci. Technol. 2013, 66, 337-344.

[28] Losego MD, Grady ME, Sottos NR, Cahill DG, Braun PV. Nat Mater. 2012, 11, 502-506. 
[29] Losego MD, Cahill DG. Nat. Mater. 2013, 12, 382-384.

[30] Nitzan A. Science 2007, 317, 759-760.

[31] Lv P, Tan XW, Yu KH, Zheng RL, Zheng JJ, Wei W. Carbon 2016, 99, 222-228.

[32] Xie YS, Xu S, Xu ZL, Wu HC, Deng C, Wang XW. Carbon 2016, 98 , 381-390.

[33] Kim HM, Noh YJ, Yu J, Kim SY, Youn JR. Composites, Part A 2015, 75, 39-45.

[34] Dorcheh AS, Abbasi M. J. Mater. Process. Technol. 2008, 199, 10-26.

[35] Patel RP, Purohit NS, Suthar AM. Int. J. Chem. Tech. Res. 2009, 1, 1052-1057.

[36] He S, Yang H, Chen X. J. Sol-Gel Sci. Technol. 2017, 82, 407-416.
[37] He S, Chen X. J. Non-Cryst. Solids 2017, 463, 6-11.

[38] Huang X, lizuka T, Jiang P, Ohki Y, Tanaka T. J. Phys. Chem. C 2012, 116, 13629-13639.

[39] Kochetov R, Korobko AV, Andritsch T, Morshuis PHF, Picken S], Smit JJ. J. Phys. D: Appl. Phys. 2011, 44, 395-401.

[40] Shahil KMF, Balandin AA. Nano Lett. 2012, 12, 861-867.

[41] Cola BA, Xu XF, Fisher TS. Appl. Phys. Lett. 2007, 90, 093513-1-3.

[42] Warzoha RJ, Zhang D, Feng G, Fleischer AS. Carbon 2013, 61, 441-457.

[43] Gong L, Kinloch IA, Young RJ, Riaz I, Jalil R, Novoselov KS. Adv. Mater. 2010, 22, 2694-2697.

[44] Jin SY, Young RJ, Eichhorn SJ. Compos. Sci. Technol. 2014, 100, 121-127. 